der nicht gedacht wird« (Dosse 2013, 349). In dem das Gefühl, ständig und immer verbunden zu sein, einen Absolutheitsanspruch erhält, verlernen wir, die Konsequenzen der algorithmischen Reduzierung der sozialen Interaktionen auf einen Marktwert zu problematisieren. Das algorithmische System ist eine Strategie zur Desorganisation der gesellschaftlichen Kräfte. Möglicherweise wird diese auch nur vorgegeben. Eine Ablehnung dieser vermeintlichen Autonomie ist elementar, sobald wir einen Eingriff oder deren tendenziöse Steuerung durch hegemoniale Akteure feststellen. Durch die Relativierung dieser Akteure können wir hoffen, dass wir als Betroffene wieder über die Bedeutung der sozialen Zukunft diskutieren können.

Die zur Allgemeinheit vorgedrungene Interaktivität erweitert die Macht von Unternehmen und einigen Staaten. Sie verbessert unsere Erfahrungen als Nutzer, wenn wir uns im Netz bewegen. Wir nutzen die Dienste und fühlen uns dabei gleichzeitig gefangen. Wir können gegenhegemoniale Alternativen ausprobieren, oder wirtschaftliche und technologische Programme hacken, die uns einschränken. Aber ob diese Aktionen wirksam sind. hängt davon $a b$, ob wir diese regimekritischen Praktiken in eine größere, komplexere Diskussion um den Sinn übertragen können. Dazu fällt mir ein Gedicht von T.S. Eliot ein:

We have the experience but missed the meaning.

And approach to the meaning restores the experience in a different form.

Die Weitergabe von Erfahrungen und ihrer Hierarchien, die von Subjekten verborgen und verarbeitet werden, welche (wie die GAFA) schon entsubjektiviert erscheinen, sollte nicht in ihrem Potenzial und ihren Gefahren bewertet werden, sondern der Erneuerung der Debatte über die Auflösung des Sinns dienen. Dabei muss allerdings klargestellt werden, dass der Sinn der Geschichte, des Lebens, des Zusammenlebens, der Konflikte und der »Lösungen«, auf den ein Dichter wie Eliot sich in der ersten Hälfte des 20. Jahrhunderts bezog, oder den Ricœur seinerzeit meinte, nur teilweise den aktuellen Erfahrungen entspricht.

\title{
Die Neuverteilung der Hegemonie
}

Ich wage folgende Hypothese über einen Sinneswandel in der sozialen Organisation und in den Erfahrungen der Bürger: der Durchbruch der sozialen Netzwerke liefert durch neue interaktive Prozesse ein Format, das jenseits 
der (kontra-)hegemonialen Absichten oder der Unternehmen und Parteien, Arten der Kommunikation und des Assoziierens eröffnet, die a priori weder hegemonial noch kontrahegemonial sind. Durch die Neukonfigurierung der Machtkonstellationen und der Akteure tauchen ambivalente und hybride Kombinationen auf. Über sie entstehen Formen der Kontaktfreudigkeit dort, wo die Macht ihre binäre Struktur verloren und eine verstreute Komplexität angenommen hat. Es gibt zahlreiche Möglichkeiten des Zusammenseins, der Kommunikation oder des Teilens und Verteilens von Gütern.

In den Netzwerken teilen wir Informationen mit Freunden und laden Fotos oder Memes hoch zur Unterhaltung oder für soziale Anerkennung. Gleichzeitig werden Nutzerressourcen abgeschöpft und oftmals veröffentlicht. Das öffentliche Teilen von geknackten Passwörtern bezeichnet man im spanischsprachigen Raum als crackear. Teilt man sie mit dem Ziel eines wirtschaftlichen Nutzens, so spricht man von Piraterie. Natürlich wird dadurch eine Köderwirtschaft etabliert, in der nach der Bezeichnung von Luis Reygadas »falsche Geschenke« an die Nutzer verteilt werden: freier Zugang oder Dienstleistungen im Gegenzug für Nutzerinformationen $(2018,74)$.

Wir erkennen, dass diese Prozesse nicht auf binäre Gegensätze reduziert werden können: Ob die Praktiken lukrativ orientiert sind oder nicht, ob legal oder illegal, kooperativ oder wettbewerbsorientiert, hegemonial oder popular. Bei einigen Interaktionen überwiegt eine Art dieser Verhaltensweisen, aber oftmals sind mehrere Akteure mit unterschiedlichen Zielen beteiligt. So wie nicht alles als Altruismus, Gegenseitigkeit und gemeinschaftlicher Aufbau in gemeinnützigen Beziehungen zu verstehen ist, $d a$ in solchen Verbindungen auch immer das Streben nach Status, Anerkennung oder Macht enthalten ist, so stützt sich die formale Wirtschaft häufig auch auf Informalität. Profitorientierte oder illegale Praktiken sind mit denjenigen aus der gleichberechtigen Zusammenarbeit verknüpft.

Wie rekonfigurieren sie also die politische Auseinandersetzung zwischen Bürgern, Unternehmen und anderen Zusammenschlüssen in der digitalen Ära? Mehrere Antworten sind hierauf möglich. Sie deuten darauf hin, dass der Begriff der Staatsbürgerschaft selbst sich verändert. Eine kritische Perspektive bietet die Diskussion zwischen Étienne Balibar und Wendy Brown. Balibar sagt darin, dass

der Neoliberalismus nicht nur eine Ideologie [ist], er ist eine Mutation der Natur der Politik selbst, die von Akteuren herbeigerufen wurde, die in allen Bereichen der Gesellschaft zu finden sind. Es handelt sich in der Tat um die 
Entstehung einer extrem paradoxen Form der politischen Aktivität. Denn er neigt nicht nur dazu, das Element der Konnektivität soweit wie möglich zu neutralisieren - was essenziell für seine klassische Erscheinung ist -, sondern er versucht, ihr im Voraus jegliche Bedeutung zu entziehen und will die Voraussetzung für eine Cesellschaft schaffen, in der Handlungen von Individuen und Gruppen (auch wenn sie gewalttätig sind) von einem einzigen Kriterium abhängen: Ihrem quantifizierbareren Nutzen. Daher geht es tatsächlich nicht so sehr um Politik oder Anti-Politik, oder um Neutralisierung oder präventive Abschaffung des soziopolitischen Antagonismus (Balibar 2013, 169).

Zur Diskreditierung von Parteien und anderen traditionellen Formen der Repräsentation kommt die Verneinung der Konfliktträchtigkeit hinzu. Balibar schreibt diese zwar dem Neoliberalismus zu, aber er neigt offensichtlich zur Verschleierung der Konflikte, die der algorithmische Reduktionismus in das soziale Leben hineinträgt. Diese entpolitisierte Perspektive kann zum blinden Vertrauen gegenüber der computerbasierten Intelligenz führen oder das Gefühl vermitteln, unsere Handlungen seien unbedeutend angesichts einer Intelligenz, die über unsere eigenen Kompetenzen hinausgeht. Dies ist bereits im Alltagsleben der Bürger und sogar in aktivistischen Kreisen eingetreten. Beim Nutzen der Netzwerke sind sie von der widersprüchlichen Informationsfülle verblüfft und sehen sich mit der Schwierigkeit konfrontiert, zwischen wahrheitsgemäßen und gefälschten Nachrichten zu unterscheiden, die von russischen oder westlichen Bots oder durch gierige journalistische Seiten für die Steigerung ihrer Klicks manipuliert wurden.

Harari warnt, dies sei erst der Anfang. Bisher begrenzen sich Tracking oder gar Spionage-Funktionen auf Webseiten nur auf externe Signale: Welche Produkte wir wo kaufen, mit wem wir kommunizieren und welche Wortwahl wir bei der Online-Suche benutzen. Doch schon bald werden biometrische Sensoren Daten über unseren Herz-Kreislauf-Rhythmus und unsere Gehirnaktivität sammeln, um sie mit den Aktivitäten unserer Kreditkarten und unseren Engagements bei Themen wie Ökologie, Jugend, Frauen und Migranten zu korrelieren. Wir sind »Tiere, die man hacken kann« (Harari 2019).

Ist politisches Handeln also nur eine Illusion und lediglich die Wirkung derer, die uns besser kennen, als wir uns selbst, und die unser Verhalten herbeiführen? Hararis Empfehlungen, um diesen Bedingungen entgegenzuwirken, sind bescheiden: a) ॥nsere Schwachpunkte kennen « - Ängste, Vorurteile, Wünsche - »denn sie sind die Hauptinstrumente derer, die sich in unser 
persönliches System einhacken wollen«; b) »die liberale Demokratie nicht nur verteidigen, weil sie bewiesenermaßen eine gütigere Regierungsform ist, als jede Alternative, sondern auch, weil sie die am wenigsten restriktive in der Debatte über die Zukunft der Menschheit ist«; c) »die traditionellen Hypothesen des Liberalismus gründlich infrage stellen und die Entwicklung eines neuen politischen Projekts stärker in einen Einklang mit den wissenschaftlichen Realitäten und den technologischen Möglichkeiten des 21. Jahrhunderts bringen« (Idem).

Wo sollen wir diese vagen Vorschläge ausüben, wenn die Rolle des Nationalstaats und generell die der territorialen Subjekte schon so weit geschrumpft ist? Anonyme Entitäten, die unsere Kommunikation tracken und wissen, wie wir auf lokaler, nationaler und globaler Ebene interagieren, wachsen weiter, aber sie generieren weder eine weltweite Gouvernementalität, noch Möglichkeiten, um sich als Bürger mit ihr verständigen $\mathrm{zu}$ können.

Vielversprechender ist hingegen die Frage nach den neuen Modi der »Souveränität« und der "algorithmischen Gouvernementalität«, und ob sie tatsächlich so global und neutral sind, wie sie vorgeben. Ist es nicht typisch für den westlichen Liberalismus, die individuellen und kollektiven Rechte $\mathrm{zu}$ verteidigen, darunter etwa die Forderung nach qualitativ hochwertiger Information? Bürger- und Regierungshandlungen gegen Google beziehen sich in manchen Fällen auf Menschenrechte, wenn es etwa um die Praxis dieses Unternehmens geht, Hassbotschaften gegen Frauen zu dulden. Sie haben Google dazu gebracht, seine Inhalte stärker zu kontrollieren. Weitere Beschwerden an Facebook wegen der Weitergabe von Falschinformationen während der Brexit-Kampagne und den Präsidentschaftswahlen in den USA zwangen die Firma letztendlich dazu, die verbreiteten Inhalte besser auszuwerten. Aus welchem Grund ist diese Art von Internet-Kontroversen in Asien weniger präsent?

Die fortgeschrittenste Annäherung an das Thema hat sich in den europäischen Ländern gezeigt. In der Folge beschloss das Europäische Parlament im April 2018 die Europäische Datenschutz-Grundverordnung. Vom 30. Mai an begann ihre Umsetzung. Unternehmen müssen ihre Valorisierung quantitativer und qualitativer Daten neu ausrichten: Das Sammeln von Informationen ist genauso wichtig wie die Art und Weise, auf diese gespeichert und verwendet werden. Die Verwaltungskultur wandelt sich angesichts der politischen und zivilgesellschaftlichen Forderungen, die mit den Forderungen nach Gewinn und den finanziellen Tricks der Konzerne in Konkurrenz stehen. 
Wenn europäische Unternehmen sich nicht an diese gesetzlichen Änderungen halten und ihre Systeme zur Verarbeitung von Kundendaten gegen die Grundverordnung verstoßen, kann sie das teuer zu stehen kommen: die Geldstrafen betragen $4 \%$ des Gesamtumsatzes, beziehungsweise bis $\mathrm{zu} 20$ Millionen Euro. Am Beispiel von Facebook haben sie gelernt, dass die Missachtung der Nutzerrechte ihren digitalen Ruf schädigen kann. Die schwachen Erfolge der Verbraucherorganisationen im medialen Zeitalter (bezogen auf Radio und Fernsehen) haben bereits die Reichweite und die Bedeutung der Staatsbürgerschaft und ihrer beginnenden Ausübung im Netz geändert. Die Anführer des E-Kapitalismus, die durch den Gang an die Börse schnell große Gewinne gemacht hatten, verloren im Juli 2018 zwischen 20 und $25 \%$ ihrer Aktienvermögen an der Wall Street. Daraufhin begannen sie, genau wie Facebook und Twitter, für eine klare Unterscheidung zwischen zuverlässigen und gefälschten Nachrichten einzustehen, um den Nutzern wieder Sicherheit zu suggerieren. Nachdem im April desselben Jahres der Datenskandal um Cambridge Analytica bekannt wurde, bestand die Notwendigkeit, der eigenen Diskreditierung schnellstmöglich entgegenzuwirken. Damals wurden zig Millionen von Nutzerdaten in sozialen Netzwerken zwecks der TrumpKampagne zweckentfremdet (»Facebook s'effondre de $18 \%$ à l'ouverture de Wall Street« 2018).

\section{Sollen tausende Silicon Valleys blühen?}

Während die transnationale Kommunikation und soziodigitale Netzwerke nationale Grenzen verschwimmen lassen, betreffen sie spezifische Regionen auf unterschiedliche Weise. Sie artikulieren sich durch wirtschaftliche und politische Auseinandersetzungen, die nicht elektronisch ablaufen. Sie bringen Bürgern in Ländern ohne fortschrittliche Technologiepolitik, darunter Lateinamerika, in eine unsicherere Lage; im Gegensatz dazu geben sie Bürgern aus europäischen Ländern mehr Möglichkeiten oder schützen sowohl deren Interessen, als auch die der dort ansässigen Unternehmen. Diese Unterschiede sind für die Diskussion in diesem Buch besonders interessant: es geht um die Reorganisation soziokultureller Aspekte der Staatsbürgerschaft. Das Fortbestehen ethnischer, nationaler und regionaler kultureller Entwicklungen, welche die angebliche Universalität des Tech-Kapitalismus widerlegen, stellt die globale Datenwirtschaft vor unterschiedliche Herausforderungen. Die lateinamerikanische Kritik am zivilisatorischen Anspruch 